\title{
Intracorporeal hemi-hand-sewn technique for Billroth-I gastroduodenostomy after laparoscopic distal gastrectomy: comparative analysis with laparoscopy-assisted distal gastrectomy
}

\author{
Yasushi Ohmura ${ }^{1,2}$, Hiromitsu Suzuki ${ }^{2,3}$, Kazutoshi Kotani ${ }^{2,4}$, Atsushi Teramoto ${ }^{2,3}$ \\ 'Department of Cancer Treatment Support Center, Okayama City Hospital, Okayama 700-8557, Japan. \\ ²Department of Surgery, Okayama City Hospital, Okayama 700-8557, Japan. \\ ${ }^{3}$ Department of Surgery, Yakage Hospital, Okayama 714-1201, Japan. \\ ${ }^{4}$ Department of Surgery, Kasaoka Daiichi Hospital, Okayama 714-0043, Japan.
}

Correspondence to: Dr. Yasushi Ohmura, Department of Cancer Treatment Support Center, Okayama City Hospital. 1-20-3 Kitanagase-omotemachi, Kita-ku, Okayama 700-8557, Japan. E-mail: yohmura826@yahoo.co.jp

\begin{abstract}
How to cite this article: Ohmura Y, Suzuki H, Kotani K, Teramoto A. Intracorporeal hemi-hand-sewn technique for Billroth-I gastroduodenostomy after laparoscopic distal gastrectomy: comparative analysis with laparoscopy-assisted distal gastrectomy. Mini-invasive Surg 2019;3:4. http://dx.doi.org/10.20517/2574-1225.2018.69
\end{abstract}

Received: 30 Nov 2018 First Decision: 30 Nov 2018 Revised: 30 Jan 2019 Accepted: 1 Feb 2019 Published: 27 Feb 2019

Science Editor: Tetsu Fukunaga Copy Editor: Cai-Hong Wang Production Editor: Huan-Liang Wu

\begin{abstract}
Aim: The purpose of this study was to evaluate the clinical feasibility and efficacy of the intracorporeal hemi-hand-sewn (IC-HHS) technique for Billroth-I gastroduodenostomy in comparison with extracorporeal total hand-sewn (EC-THS) anastomosis. We also examined the size of resected specimens in each procedure.
\end{abstract}

Methods: The number of enrolled cases of EC-THS and IC-HHS anastomosis groups were 85 and 110 cases, respectively. Perioperative data and the measured sizes of resected specimens were analyzed.

Results: Operation time in the IC-HHS group was significantly longer than the EC-THS group (234.8 min vs. 275.0 min, $P<0.01)$, whereas intraoperative blood loss was less in the IC-HHS group $(48.4 \mathrm{~mL}$ vs. $25.4 \mathrm{~mL}, P=0.03$ ). There were no procedure-related complications in the IC-HHS group. The greater curvature of the EC-THS group was significantly shorter than the IC-HHS group ( $214.6 \mathrm{~mm} v$ s. $228.7 \mathrm{~mm}, P<0.01$ ). There was no correlation between body mass index (BMI) and the length of the greater curvature in the IC-HHS group $(r=0.07, P=0.47)$, but in the EC-THS group, the length of the greater curvature tends to shorten as BMI increases $(r=-0.45, P<0.01)$.

Conclusion: IC-HHS technique for Billroth-I gastroduodenostomy revealed feasible with acceptable operation time and postoperative outcome. Another advantage of total laparoscopic distal gastrectomy that intracorporeal transection can facilitate is to ensure an adequate proximal margin, especially in obese middle gastric cancer patients.

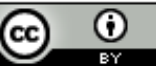

(C) The Author(s) 2019. Open Access This article is licensed under a Creative Commons Attribution 4.0 International License (https://creativecommons.org/licenses/by/4.0/), which permits unrestricted use, sharing, adaptation, distribution and reproduction in any medium or format, for any purpose, even commercially, as long as you give appropriate credit to the original author(s) and the source, provide a link to the Creative Commons license, and indicate if changes were made.

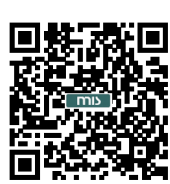


Keywords: Laparoscopy, gastrectomy, anastomosis, Billroth I procedure, surgical margin

\section{INTRODUCTION}

Since the first laparoscopic gastrectomy was described in $1992^{[1]}$, the safety and feasibility of laparoscopic gastrectomy have already been demonstrated in the treatment of early gastric cancer ${ }^{[2,3]}$ and several advantages of laparoscopic gastrectomy were reported in comparison with open gastrectomy, such as postoperative recovery and shorter hospital stay ${ }^{[4,5]}$. Due to being less invasive, laparoscopic gastrectomy was successfully performed in elderly patients and obese patients with an acceptable complication rate and $\operatorname{prognosis}^{[6-9]}$.

The reconstruction methods after distal gastrectomy are represented by Billroth-I (B-I), Billroth-II, and Roux-en-Y method. Among them, B-I gastroduodenostomy is the most widely practiced procedure in the world. Due to the fact that the procedure is relatively simple and does not require an anatomical replacement of the digestive tract below the transverse $\operatorname{colon}^{[10]}$. Moreover, it provides a physiologic flow of food contents through the duodenum and decreases the possibility of metabolic problems and nutritional deficiency ${ }^{[11]}$ while postoperative observation of the ampulla of Vater can be carried out reliably and easily. When laparoscopic gastrectomies were first introduced, because of the technical challenges of achieving an intracorporeal B-I reconstruction, most surgeons preferred laparoscopy-assisted approach with minilaparotomy ${ }^{[12,13]}$. In laparoscopy-assisted distal gastrectomy (LADG), gastroduodenostomy as well as gastric transection was also performed through a small laparotomy. Therefore, especially in obese patients with thick abdominal walls, it was difficult to pull out the stomach enough to secure an appropriate resection range and to perform safe anastomosis through the small laparotomy.

Recently, several techniques of intracorporeal reconstruction have been developed. Currently, many gastric surgeons are attempting to perform total laparoscopic gastrectomy with intracorporal reconstruction because it offers a good operative field regardless of the patient's figure ${ }^{[14]}$. Furthermore, the transection level of the stomach can be reliably determined by the intraoperative endoscope in a natural anatomical position without deformation.

In our hospital, we have performed LADG with extracorporeal total hand-sewn (EC-THS) gastroduodenostomy through mini-laparotomy until October $2013^{[15]}$. Since intracorporeal delta-shaped (IC-DS) gastroduodenostomy was introduced, this method became the most widely implemented intracorporeal anastomotic technique, especially in eastern countries ${ }^{[16-18]}$. However, several concerns have been reported, such as anatomical twisting, excessive tension caused by side-to-side anastomosis, duodenal ischemia due to duodenal dissection and preservation, and shortened distal surgical margin. In 2013, as the method of total laparoscopic distal gastrectomy, we devised a new reconstruction method to create end-to-end gastroduodenostomy, in which the posterior wall of the anastomosis was constructed with a linear stapler and subsequently, the anterior wall was sutured with an intracorporeal hand-sewn technique, and reported as intracorporeal "hemi-hand-sewn (HHS) technique" ${ }^{\text {"[19] }}$.

The purpose of this study was to evaluate the feasibility and efficacy of IC-HHS technique for end-to-end B-I gastroduodenostomy after laparoscopic distal gastrectomy in comparison with conventional EC-THS anastomosis in LADG. In addition, we assumed that there was a possibility that the range of resection might be smaller in laparoscopy-assisted approach in obese patients, but the difference of resection range between the extracorporeal transection and intracorporeal procedure were not reported. Therefore, we evaluated the size of resected specimen and analyzed the differences of the resection range between each procedure. 


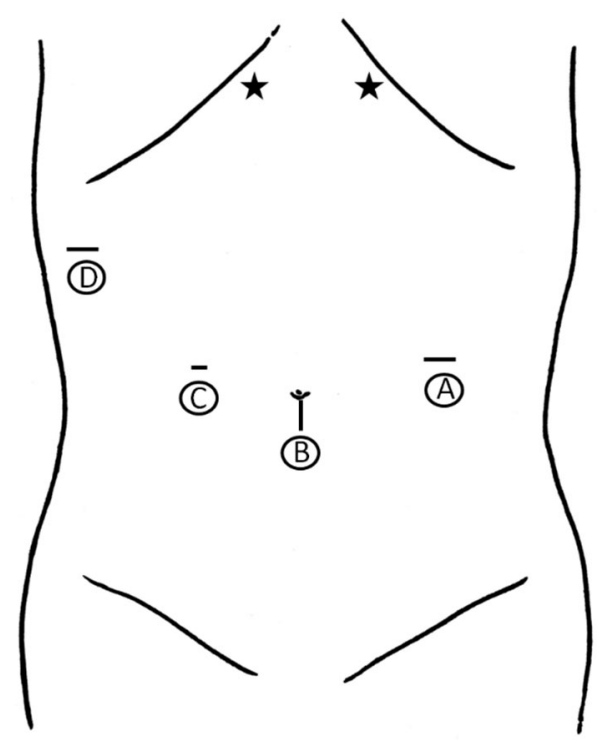

Figure 1. The trocar placement and stomach lifting positions for dual stomach-lifting technique in total laparoscopic distal gastrectomy. All trocars are $12 \mathrm{~mm}$. A and $C$ are used by the operator and D by the assistant. A flexible laparoscope is inserted through B. The nylon threads for stomach lifting are pulled out from the star marks

\section{METHODS}

\section{Lymph node dissection}

Under general anesthesia, the patient was placed in a modified lithotomy position and subjected to a head-up tilt with the semiboot foot holder: Levitator (Mizuho, Tokyo, Japan). We used a 10-mm, 3D flexible laparoscope; ENDOEYE FLEX 3D ${ }^{\oplus}$ (Olympus Medical, Tokyo, Japan). The operator performed all procedures standing between the patient's legs. We applied a 4 port-laparoscopic distal gastrectomy with dual stomach-lifting technique (DSLT) ${ }^{[15]}$. The trocar placement and stomach lifting positions are shown in Figure 1. The stomach and duodenum were not transected before completion of the lymph node dissection.

\section{Gastric resection and EC-THS gastroduodenostomy in LADG}

Endoscopic clips were preoperatively placed on the proximal site $1 \mathrm{~cm}$ from the tumor along the longitudinal axis of the stomach. In the EC-THS group, the mobilized stomach was pulled out via Lap Protector $^{\circledast}$ (Hakko, Nagano, Japan) attached to a 5-cm epigastric mini-laparotomy. After confirmation of clips by palpation, the distal stomach was divided from the greater curvature side by a linear stapler extracorporeally. Then staple line of the remnant stomach was reinforced by continuous seromuscular suturing with 3-0 coated braided synthetic absorbable suture: Vicryl ${ }^{\circledR}$ (Ethicon, Somerville, NJ, USA) from the lesser curvature. Then posterior sero-muscular suturing between the remnant stomach and the duodenum were performed with 3-0 coated braided nylon suture: Bear Braid ${ }^{\circledast}$ (Bear Medic Co., Tokyo, Japan). After ligation, the duodenum was divided by electric scalpel and the distal gastrectomy was completed. When the proximal edge of the tumor was close to the resection margin, frozen-section examination was performed to confirm the absence of any microscopic invasion. Subsequently, wholelayer continuous sutures of the posterior and anterior wall were performed with 3-0 Vicryl. Finally, 2-layer gastroduodenostomy was completed after sero-muscular suture with 3-0 Bear Braid.

\section{Gastric resection and IC-HHS gastroduodenostomy in TLDG}

Preoperative endoscopic marking was done with the same maneuver as LADG. Lymph node dissection was also performed with DSLT. Details of surgical procedures and the IC-HHS technique were summarized in the attached video [Video 1]. 
Endoscopic observation to the duodenum prior to resection made it possible to prevent spillage of gastrointestinal contents during anastomosis. After completion of lymph node dissection, the duodenum was divided with an endoscopic linear stapler: Echelon 60-3.5 (Ethicon Endo-Surgery, Cincinnati, OH, USA) without duodenal twisting. Transection of the stomach was also performed using endoscopic linear staplers twice under intraoperative gastroscopic navigation (in middle third gastric cancer cases). Then the resected specimen was retrieved via Lap Protector ${ }^{\circledast}$ (Hakko, Nagano, Japan) attached to a $3-\mathrm{cm}$ incision at the umbilical port. After the macroscopic evaluation, a frozen-section examination was performed if needed.

\section{Full-thickness posterior wall anastomosis}

After retrieval of the resected stomach, a plate platform: E-Z access (Hakko) was attached to the wound protector and pneumoperitoneum was restarted, then an intracorporeal anastomosis was performed. First, removal of a small part of the staple using Sonicbeat ${ }^{\circ}$ (OLYMPUS, Tokyo, Japan) at $5 \mathrm{~cm}$ from the greater curvature of the remnant stomach was completed to confirm anastomotic diameter. Then the staple line of the remnant stomach was reinforced by continuous seromuscular suturing with 3-0 Vicryl from the lesser curvature to the defect of the staple. Sero-muscular stay sutures with 3-0 Bear Braid were placed at both the lesser and greater curvatures between the remnant stomach and the duodenum. An entry hole was made by piercing the active blade of Sonicbeat at the greater curvature of the stomach wall, then a tissue pad of Sonicbeat was put into the stomach cavity and the anterior wall was incised toward the staple defect. A similar entry hole was made at the greater curvature of the duodenal wall and the anterior wall of the duodenum was incised toward the lesser curvature side. The assistant grasped two stay sutures ligated at the excessive tissues and lifted them up vertically. Then the posterior wall of the remnant stomach and the duodenum were approximated by Echelon 60-3.5 inserted from the umbilical port. During closure of the stapler forks, it was carefully confirmed that there was no excessive pinching of the anterior wall from left and right lateral abdominal trocars. The endoscopic linear stapler was fired to excise the excessive gastric and duodenal tissues and simultaneously the posterior wall anastomosis was constructed. Then the staple line was observed to confirm that there was no bleeding or pinching of the anterior wall [Figure $2 \mathrm{~A}-\mathrm{G}$ ].

\section{Full-thickness anterior wall anastomosis}

Subsequently, a full-thickness, continuous suture of the anterior wall was performed with 3-0 Vicryl. The first suture was started at the lower edge of the posterior wall and the first knot was developed inside the lumen involving the staple edge. Next suturing was made from the duodenal mucosa to the serosal side and after 5 over and over suturings, the Vicryl was locked. Using another $20 \mathrm{~cm}$ Vicryl, continuous suturing was started from the upper edge of the staple line of the posterior wall anastomosis. Continuous suturing was carried out with an over and over technique and when the two sutures met each other, each end was ligated intracorporeally to finish the full-thickness anterior wall anastomosis [Figure $2 \mathrm{H}$ ].

\section{Sero-muscular inverting anterior wall anastomosis}

Afterwards, interrupted sero-muscular layer suturing of the anterior wall was performed with 3-0 Bear Braid for complete inverting anastomosis [Figure 2I]. The first suture at the lesser curvature involved three points, the anterior and posterior wall of the remnant stomach and the duodenal wall. Approximately 10 sero-muscular sutures were required for inverting gastroduodenostomy. Then, an intracorporeal B-I gastroduodenostomy by HHS technique was completed, which was almost the same shape as a handsewn inverting anastomosis by open surgery or EC-THS reconstruction. In the case with intraoperative endoscopy, the anastomotic site was observed after the completion of reconstruction [Figure 3].

\section{Patient evaluation}

Total laparoscopic gastrectomy with IC-HHS technique was introduced since November 2013. A total of 452 patients who underwent surgery for gastric cancer in our hospital and related institutions between September 2008 and December 2017 were identified. All patients underwent multi-detector-row computed 

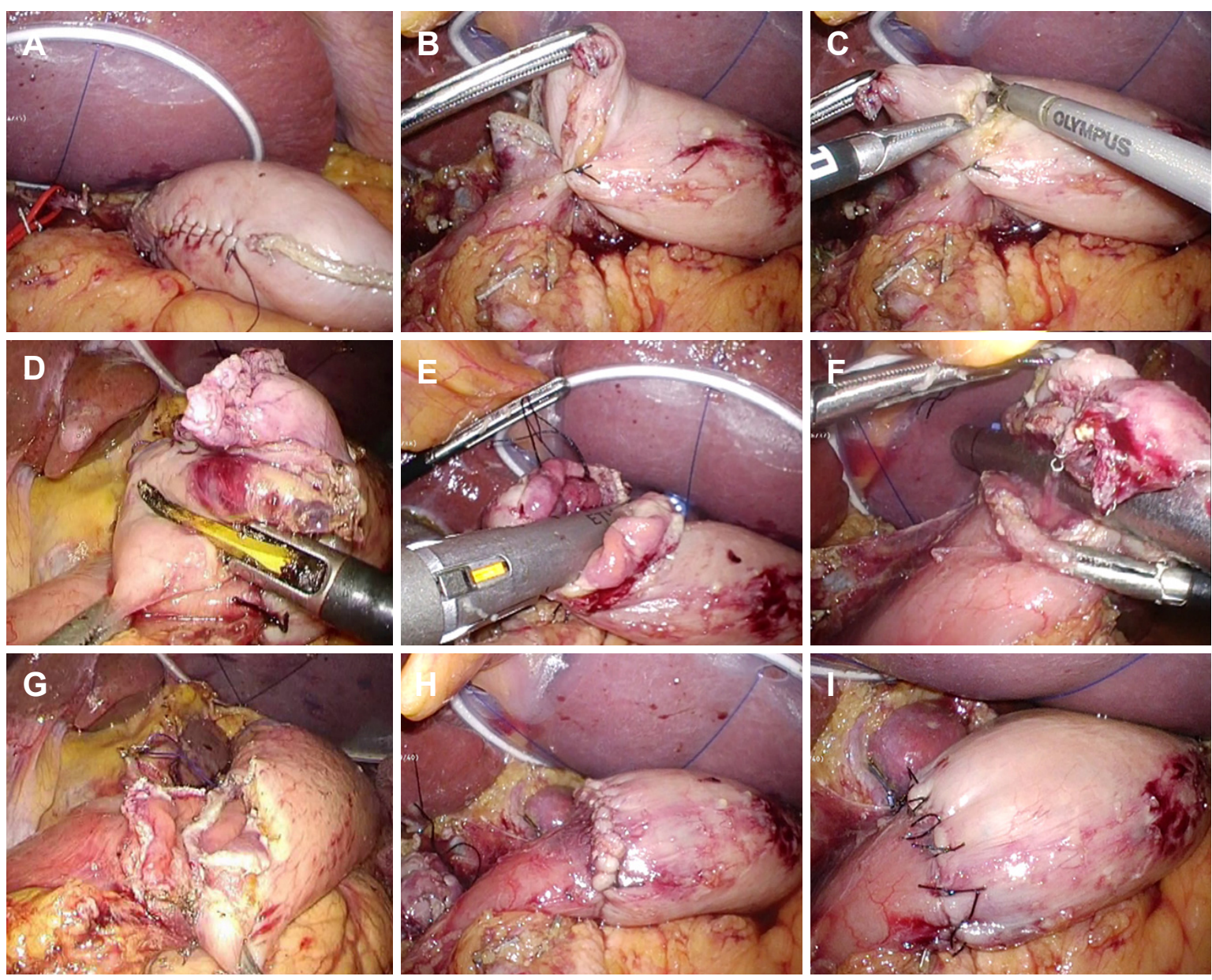

Figure 2. Intracorporeal hemi-hand-sewn Billroth-I anastomosis. After having confirmed approximately $5 \mathrm{~cm}$ away from the edge of the greater curvature for anastomosis, the remaining staple line is reinforced by continuous suture (A); sero-muscular stay sutures are placed between the remnant stomach and the duodenum (B); the anterior wall of the remnant stomach is incised (C); subsequently, the anterior wall of the duodenum is also incised (D); the posterior wall of the remnant stomach and the duodenum is brought together with the Echelon 60-3.5 from the umbilical port: laparoscopic view from the stomach side (E) and from the duodenal side (F); full thickness of posterior wall anastomosis is completed (G); then a whole-layer approximation is accomplished by continuous suture with an absorbable thread $(\mathrm{H})$; finally, an anterior sero-muscular suture is completed by interrupted sutures (I)

tomography scans and upper endoscopy to determine the location and clinical stage of gastric cancer. Adenocarcinoma of the stomach was histologically proven for all patients. Open gastrectomy was performed in 229 patients, while 223 patients underwent laparoscopic gastrectomy. In this study, 21 total gastrectomy cases and 3 proximal gastrectomy cases were excluded. Laparoscopic distal gastrectomy was performed in 199 cases. Among them, 3 patients underwent concurrent colectomy and 1 case with concurrent nephrectomy were also excluded. A total of 195 patients were enrolled into the retrospective study [Figure 4]. All patients gave their written informed consent for laparoscopic procedures. This study protocol was approved by the institutional ethics committee. Demographic characteristics such as age, sex, body mass index (BMI), ASA score, site of the lesion, history of abdominal surgery, and anticoagulant use, were retrospectively extracted from the database.

\section{Surgical outcome and postoperative complications}

Operation time, blood loss, extent of lymph node dissection, number of harvested lymph nodes, pathological stage, perioperative complications, length of hospital stay after surgery, and type of reconstruction were retrieved from the database. Postoperative complications were graded according to the Clavien-Dindo classification ${ }^{[20]}$. Delayed gastric emptying was defined as an emptying disturbance 


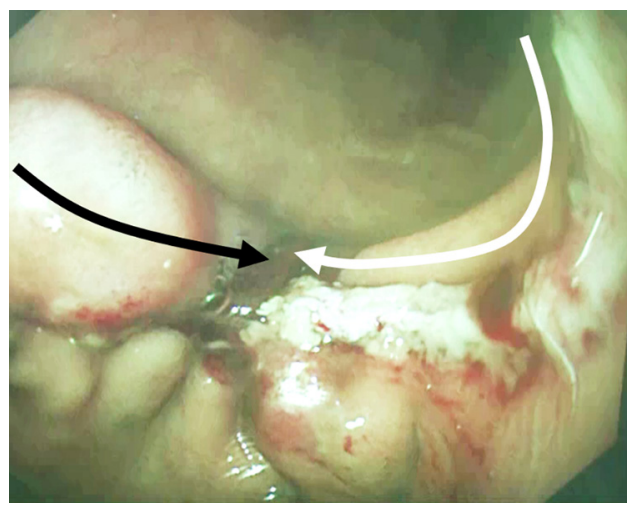

Figure 3. Intraoperative endoscopic finding after completion of gastroduodenostomy by intracorporeal hemi-hand-sewn technique. The suture line of the posterior wall by linear stapler (white arrow) and hand-sewn suture line of the anterior wall (black arrow)

requiring starvation for more than three days, excluding the cases where anastomotic stenosis or mechanical bowel obstruction was confirmed by radiographic or endoscopic examination.

\section{Evaluation of resected specimen}

The resected stomach was incised and opened according to the rule of Japanese classification of gastric carcinoma $^{[21]}$ and then was placed on the flat board. After stretching the stomach wall sufficiently, we fixed the edges of the stomach to the board with stainless steel pins. In addition to measuring the size of the tumor, we also measured the length of the greater curvature, lesser curvature, duodenum, proximal margin and distal margin. Where the staple line was removed, a length of $3 \mathrm{~mm}$ was added to each as a measured value. Histological data were retrieved from the database based on the 14th version of the staging system of Japanese Gastric Cancer Association ${ }^{[21]}$.

\section{Statistical analysis}

Clinicopathological data and perioperative results were statistically compared between the EC-THS group and the IC-HHS group. The length of each part of the resected specimen was also analyzed. Continuous data was analyzed with the Student's $t$-test. The Fisher's exact test or Chi-square test were used for comparison of categorical values. Pearson linear regression analyses were conducted to correlate BMI and the length of the greater curvature of resected stomach. A two-sided $P$ value $<0.05$ was considered to be statistically significant.

\section{RESULTS}

\section{Patient characteristics}

The number of cases of the EC-THS and IC-HHS groups were 85 and 110 cases, respectively. The 195 patients had an average age of 71.0 years (range, 38-93 years). The average BMI was 23.1 (range 17.0-34.0 kg/m²). As for the location of the tumors, 142 cases were in the middle third of the stomach and 53 cases was in the lower third of the stomach, 5 of them showed duodenal infiltration to the pyloric ring [Table 1].

\section{Surgical outcome}

Total laparoscopic or laparoscopy-assisted distal gastrectomies were successfully accomplished in all patients (195 patients). D2 lymph node dissection was performed more frequently in the IC-HHS group (12.7\%) compared to the EC-THS group $(1.2 \%)(P<0.01)$. The proportion of concurrent cholecystectomy was higher in the IC-HHS group, but there was no significant difference $(P=0.21)$. Operation time in the IC-HHS group required an average of $275.0 \mathrm{~min}$, which was significantly longer than the $234.8 \mathrm{~min}$ in the EC-THS group $(P<0.01)$. The amount of intraoperative blood loss was less in IC-HHS group $(25.4 \mathrm{~mL}$ vs. $44.0 \mathrm{~mL}, P=0.03)$. The subgroup analysis in $\mathrm{D} 1+$ cases revealed similar results; the average operation 


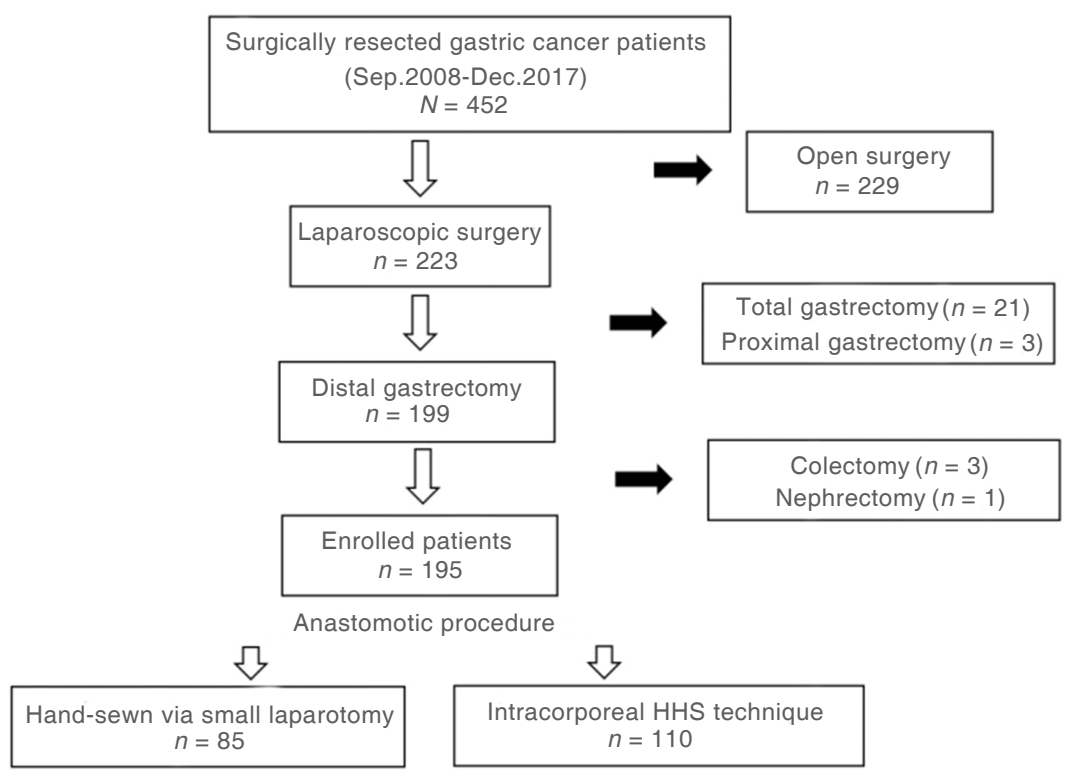

Figure 4. Flow chart for the current study detailing inclusion and exclusion criteria; HHS: hemi-hand-sewn

time was longer in the IC-HHS group (276.4 $\mathrm{min})$ than in the EC-THS group $(234.6 \mathrm{~min})(P=0.04)$ and intraoperative blood loss was smaller in IC-HHS group $(26.3 \mathrm{~mL})$ compared to EC-THS group (48.9 $\mathrm{mL})$ $(P<0.01)$. Ro resection was achieved in all patients with free surgical margin defined by pathological examination.

\section{Postoperative outcome}

Surgical site infection was observed at the epigastric wound in the EC-THS group (5 cases) and at the umbilical wound in the IC-HHS group (1 case). Laparoscopic cholecystectomy was required in one case of the IC-HHS group due to necrotic cholecystitis resistant to conservative treatment on day 9 after gastrectomy. One case of delayed gastric emptying was observed in the EC-THS group. The frequency of intra-abdominal abscess or pancreatic fistula development in the EC-THS and IC-HHS group were 3.5\% and $1.8 \%$, respectively and they were all cured by administration of antibiotics. One case of postoperative pneumonia in the EC-THS group was also successfully treated by antibiotics.

\section{Evaluation of resected specimen}

Table 2 shows the measured length of each part of the resected specimens. The greater curvature of the ICHHS group was significantly longer than that of EC-THS group (214.6 vs. $228.7 \mathrm{~mm}, P<0.01)$. The length of the lesser curvature and the duodenum were not significantly different. There was no correlation between BMI and the length of the greater curvature in the IC-HHS group $(r=0.03, P=0.47)$, while in the EC-HS group, the length of the greater curvature weakly correlated with BMI $(r=-0.44, P<0.01)$. The length of the great curvature of the resected stomach tends to be shorter as BMI increases [Figure 5A and B].

\section{DISCUSSION}

This study found that the IC-HHS technique for laparoscopic B-I gastroduodenostomy was successfully accomplished in all 110 cases without any procedure-specific complications, indicating its technical feasibility. The average operation time was about 40 min longer than EC-THS reconstruction. The similar difference in the subgroup analysis in D1+ cases suggested that the extended operation time was due to the difference in the anastomotic procedures. Anastomotic leakage and stricture were not observed in our experiences. 
Table 1. Demographic characteristics, perioperative details, and pathological outcome of gastric cancer patients

\begin{tabular}{|c|c|c|c|c|c|}
\hline \multirow{2}{*}{ Variable } & \multicolumn{2}{|c|}{ EC-THS $(n=85)$} & \multicolumn{2}{|c|}{ IC-HHS $(n=110)$} & \multirow{2}{*}{$P$ value } \\
\hline & Mean \pm SD & Range & Mean \pm SD & Range & \\
\hline \multicolumn{6}{|l|}{ Backgrounds } \\
\hline Age, mean $\pm S D(y . o)$. & $70.7 \pm 9.5$ & $44-88$ & $71.2 \pm 10.2$ & $38-93$ & $P=0.68$ \\
\hline \multicolumn{6}{|l|}{ Sex, No. (\%) } \\
\hline Male & $58(68.2 \%)$ & & $77(70.0 \%)$ & & $P=0.79$ \\
\hline Female & $27(31.8 \%)$ & & $33(30.0 \%)$ & & \\
\hline $\mathrm{BMI}$, mean $\pm \mathrm{SD}\left(\mathrm{kg} / \mathrm{m}^{2}\right)$ & $23.5 \pm 3.3$ & $19-31$ & $22.7 \pm 3.5$ & $17-34$ & $P=0.17$ \\
\hline \multicolumn{6}{|l|}{ ASA classification, No. (\%) } \\
\hline I & $17(20.0 \%)$ & & $18(16.4 \%)$ & & $P=0.57$ \\
\hline II & $55(64.7 \%)$ & & $74(67.3 \%)$ & & \\
\hline III & $13(15.3 \%)$ & & $18(16.4 \%)$ & & \\
\hline \multicolumn{6}{|l|}{ Location, No. (\%) } \\
\hline Upper stomach & $0(0.0 \%)$ & & $0(0.0 \%)$ & & $P=0.21$ \\
\hline Middle stomach & $58(68.2 \%)$ & & $84(76.4 \%)$ & & \\
\hline Lower stomach & $27(31.8 \%)$ & & $26(23.6 \%)$ & & \\
\hline Lower stomach $(\mathrm{D}+)$ & $2(1.8 \%)$ & & $3(2.7 \%)$ & & \\
\hline \multicolumn{6}{|l|}{ Prior abdominal surgery, No. (\%) } \\
\hline Yes & $23(27.1 \%)$ & & $20(18.2 \%)$ & & $P=0.14$ \\
\hline No & $62(72.9 \%)$ & & $90(81.8 \%)$ & & \\
\hline \multicolumn{6}{|l|}{ Anticoagulant use, No. (\%) } \\
\hline Yes & $17(20.0 \%)$ & & $21(19.1 \%)$ & & $P=0.87$ \\
\hline No & $68(80.0 \%)$ & & $89(80.9 \%)$ & & \\
\hline \multicolumn{6}{|l|}{ Perioperative details } \\
\hline \multicolumn{6}{|l|}{ Lymph node dissection, No. (\%) } \\
\hline $\mathrm{D} 1+$ & $84(98.8 \%)$ & & $96(87.3 \%)$ & & $P<0.01$ \\
\hline D2 & $1(1.2 \%)$ & & $14(12.7 \%)$ & & \\
\hline Operation time, mean $\pm \mathrm{SD}(\mathrm{min})$ & $234.8 \pm 29.6$ & $175-302$ & $275.0 \pm 34.7$ & $230-411$ & $P<0.01$ \\
\hline Blood loss, mean $\pm \mathrm{SD}(\mathrm{mL})$ & $48.4 \pm 39.6$ & $5-180$ & $25.4 \pm 32.6$ & $3-140$ & $P=0.03$ \\
\hline Cholecystectomy, No. (\%) & $8(9.4 \%)$ & & $17(15.6 \%)$ & & $P=0.21$ \\
\hline \multicolumn{6}{|l|}{ Complications, C-D grade No. (\%) } \\
\hline Grade I & $5(5.9 \%)$ & & $1(0.9 \%)$ & & \\
\hline Grade II & $4(4.7 \%)$ & & $2(1.8 \%)$ & & \\
\hline Grade IIla & $0(0.0 \%)$ & & $0(0.0 \%)$ & & \\
\hline Grade IIIc & $0(0.0 \%)$ & & $1(0.9 \%)$ & & \\
\hline $\mathrm{LOHS}$, mean $\pm \mathrm{SD}$ (day) & $14.6 \pm 3.8$ & $5-23$ & $13.3 \pm 4.8$ & $7-34$ & $P=0.04$ \\
\hline \multicolumn{6}{|l|}{ Pathological outcomes } \\
\hline \multicolumn{6}{|l|}{ T stage, No. (\%) } \\
\hline Mucosa & $47(55.3 \%)$ & & $64(58.2 \%)$ & & $P=0.83$ \\
\hline Submucosa & $24(28.2 \%)$ & & $27(24.5 \%)$ & & \\
\hline Muscularis propria & $8(9.4 \%)$ & & $8(7.3 \%)$ & & \\
\hline Subserosa & $6(7.1 \%)$ & & $11(10.0 \%)$ & & \\
\hline \multicolumn{6}{|l|}{ N stage, No. (\%) } \\
\hline 0 & $83(97.6 \%)$ & & $105(95.5 \%)$ & & $P=0.43$ \\
\hline $1(1-3)$ & $2(2.4 \%)$ & & $5(4.5 \%)$ & & \\
\hline Lymph node yield, mean $\pm \mathrm{SD}$ (No.) & $30.8 \pm 10.2$ & $11-59$ & $29.4 \pm 9.8$ & $11-59$ & $P=0.34$ \\
\hline \multicolumn{6}{|l|}{ Pathological stage, No. (\%) } \\
\hline IA & $67(78.8 \%)$ & & $90(81.8 \%)$ & & $P=0.75$ \\
\hline IB & $11(12.9 \%)$ & & $8(7.3 \%)$ & & \\
\hline$\| A$ & $6(7.1 \%)$ & & $6(5.5 \%)$ & & \\
\hline IIB & $1(1.2 \%)$ & & $6(5.5 \%)$ & & \\
\hline
\end{tabular}

EC-THS: extracorporeal-total hand-sewn group; IC-HHS: intracorporeal-hemi-hand-sewn group; SD: standard deviation; BMI: body mass index; ASA score: American Society of Anthologist score; C-D grade: Clavien-Dindo classification grade; LOHS: post-operative length of hospital stay

Though a B-I gastroduodenostomy requires only one anastomosis, it is difficult to perform through a small laparotomy in obese patients. The degree of difficulty is greatly affected by the patient's figure. On the 
A Length of the grater curvature

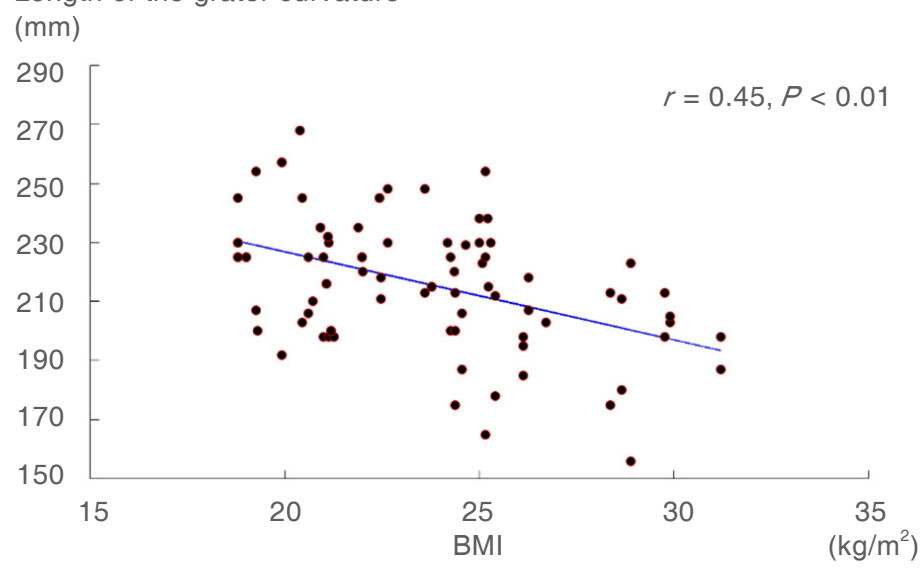

B Length of the grater curvature

$(\mathrm{mm})$

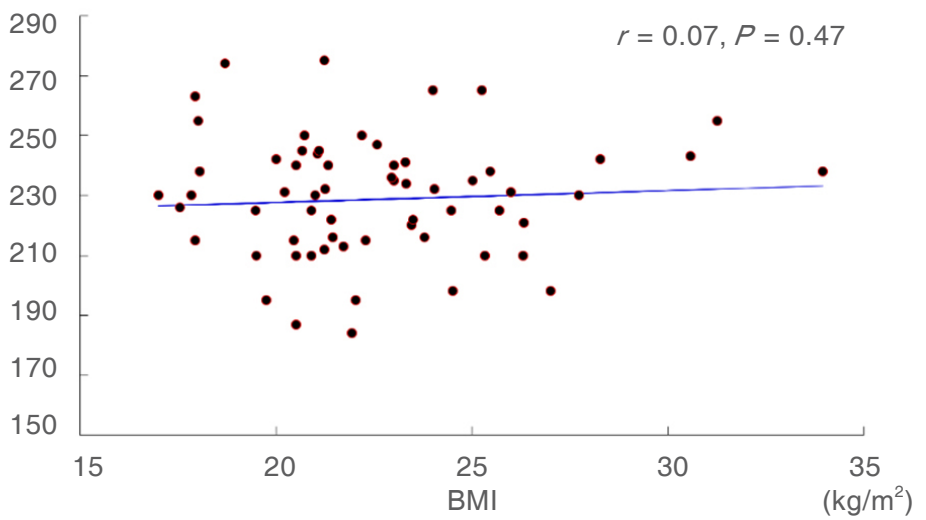

Figure 5. Simple linear regression analyses between $\mathrm{BMI}$ and the length of the greater curvature of resected stomach. The length of the greater curvature weakly correlated with BMI in the extracorporeal total hand-sewn group (A); while there was no correlation between $\mathrm{BMI}$ and length of the greater curvature in the intracorporeal hemi-hand-sewn group (B)

Table 2. Size of resected specimen

\begin{tabular}{lccc}
\hline & EC-THS $(\boldsymbol{n}=\mathbf{8 5})$ & IC-HHS $(\boldsymbol{n}=\mathbf{1 1 0})$ & $\boldsymbol{P}$ value \\
\hline Maximum tumor, mean \pm SD $(\mathrm{mm})$ & $31.6 \pm 15.5$ & $35.6 \pm 28.1$ & $P=0.20$ \\
Greater curvature, mean \pm SD (mm) & $214.6 \pm 21.7$ & $228.7 \pm 19.7$ & $P<0.01$ \\
Lesser curvature, mean \pm SD $(\mathrm{mm})$ & $148.7 \pm 17.6$ & $149.2 \pm 18.3$ & $P=0.50$ \\
Duodenum, mean \pm SD $(\mathrm{mm})$ & $14.7 \pm 3.6$ & $14.1 \pm 4.0$ & $P=0.22$ \\
Proximal margin, mean \pm SD (mm) & $53.4 \pm 32.4$ & $54.1 \pm 32.5$ & $P=0.88$ \\
Distal margin, mean \pm SD $(\mathrm{mm})$ & $77.1 \pm 35.7$ & $78.5 \pm 39.8$ & $P=0.87$ \\
\hline
\end{tabular}

SD: standard deviation; EC-THS: extracorporeal-total hand-sewn group; IC-HHS: intracorporeal-hemi-hand-sewn group

other hand, intracorporeal B-I reconstruction by means of open surgery requires considerable advanced technical skill. Therefore, several procedures for B-I intracorporeal gastroduodenostomies specific to laparoscopic surgery have been reported, such as IC-DS gastroduodenostomy ${ }^{[16]}$, triangulation stapling technique ${ }^{[22]}$, book binding technique ${ }^{[23,24]}$, overlap method ${ }^{[25]}$, and double-stapling technique with circular stapler. Sufficient blood supply of digestive tracts, absence of excessive tension, well-apposed tissues at the anastomotic site, and appropriate anastomotic techniques are critically important factors for successful and safe anastomosis. In terms of blood supply and tension between the reconstructing digestive tract, hemidouble stapling technique with circular stapler is the best procedure for gastroduodenostomy ${ }^{[26]}$. However, 
it is difficult to keep a sufficient operative field for safe intracorporeal anastomosis. Currently, IC-DS anastomosis has been widely performed in East Asian countries ${ }^{[14,16]}$. Shorter operation time and superior short-term surgical outcomes were reported in comparison with the other methods ${ }^{[27,28]}$, however, some concerns were reported. First, due to anatomical twisting from the IC-DS method, it is relatively difficult for inexperienced surgeons to understand deviated anatomy. Second, a IC-DS gastroduodenostomy is a functional end-to-end anastomosis, but is an anatomical side-to-side anastomosis, more tension is generated compared to anatomical end-to-end anastomosis ${ }^{[23]}$. Moreover, additional duodenal dissection and formation of the triangular area of the duodenal wall might be responsible for the ischemic status, which is one of the most significant risk factors of anastomotic failure. Technically, a skillful assistant is desirable for cooperative work when inserting a stapler during IC-DS gastroduodenostomy. Okabe et al. ${ }^{[29]}$ reported two cases of duodenal injury during IC-DS gastroduodenostomies and they had to change the reconstruction method.

On the other hand, other anastomotic procedures using intracorporeal hand-sewn techniques were reported ${ }^{[30-32]}$. Takiguchi et al..$^{[30]}$ performed a total laparoscopic method by performing Billroth-I handsewn anastomosis and reported excellent postoperative recovery. In attempting to intracorporeal anastomosis, we initially applied total hand-sewn Albert-Leinbert anastomosis which was a promising and stable anastomotic method in open surgery after thorough training and since 2012. Total hand-sewn Albert-Leinbert anastomosis was performed in 19 patients. Although there was no major problem in their postoperative course, it took an average of nearly 100 min longer compared to the EC-THS method. Then, we devised a new reconstruction method to create end-to-end gastroduodenostomy, in which the posterior wall of the anastomosis was constructed with a linear stapler and subsequently, the anterior wall was sutured by an intracorporeal hand-sewn technique. We previously reported this procedure as a "HHS technique" in 2013 ${ }^{[19]}$. Koeda and colleagues reported similar technique as a hybrid technique using linear staplers and manual suturing in 19 pylorus-preserving gastrectomy cases with good postoperative results ${ }^{[33]}$.

There are some reports using a barbed suture in order to facilitate intracorporeal suturing, however, complications following the use of this unique suture have been reported in several other surgeries ${ }^{[34-36]}$. The most commonly encountered complication with the use of barbed sutures was postoperative bowel obstruction. In terms of its intended shape, a barbed suture develops irreversible tension created during the suturing. Therefore, excessive traction narrows the anastomotic lumen, which can also cause postoperative stricture. Therefore, we used absorbable blade for continuous Albert suturing and nylon blade for interrupted Leinbert suturing, as were adopted to conventional open surgery.

Although single layer continuous anastomosis could simplify the procedure and shorten operation time, we performed two-layer anastomosis. In bariatric surgery, staple-line reinforcement was strongly recommended for laparoscopic sleeve gastrectomy to decrease complications ${ }^{[37]}$. Also, it was reported that laparoscopic reinforcement with Lembert's sutures of a duodenal stump could help to avoid duodenal stump leakage in Rou-en Y reconstruction ${ }^{[38,39]}$. We applied a two-layer technique with interrupted inverting sutures for the intracorporeal gastroduodenostomy. Due to the fact laparoscopic surgery has been developed as a minimally invasive surgery, once postoperative complications arise, the disadvantages incurred by patients are greater than that of conventional laparotomy surgery. Reconstruction can be done without hand suturing by other anastomotic techniques in most cases, however, there are no reports showing the result of delta-shaped anastomosis in open gastrectomy even at medical facility that introduced IC-DS anastomosis as the standard procedure. This does not mean that delta anastomosis is the best anastomotic method but may indicate that it is a complementary procedure during the development of laparoscopic surgery. We believe that acquisition of intracorporeal suturing skills are still important and the ideal reconstruction methods established in the long history of open surgery deserved to be reproduced as much as possible in laparoscopic surgery. IC-HHS anastomosis requires an advanced suturing technique 
and longer operation time, but we believe it is undesirable to give too much priority to shortening operation time. Completing a straight suture line in gastroduodenostomy at the co-axial position is a great opportunity to acquire basic intracorporeal suturing technique and the acquired technique will be useful in unexpected difficult situations, such as unintended bowel injury.

In LADG, extracorporeal stomach transection with a linear stapler is performed from the greater curvature and it is sometimes difficult to ensure an optimal proximal margin in obese patients with middle third gastric cancer. When the patient has a thick abdominal wall, it is necessary to pull out the stomach with considerable traction however there is an absolute limitation to our ability. At the beginning of the totally laparoscopic distal gastrectomy (TLDG), there were some concerns about the difficulty of locating the tumor and securing the resection margin as a technical limitation. However, with the routine intraoperative endoscopy, it has become possible to decide the transection line more reliably. Although there were some reports analyzing the data of proximal and distal resection $\operatorname{margin}^{[40-43]}$, the difference of the resected specimen between extracorporeal and intracorporeal approach had not been discussed. Therefore, we conducted an evaluation of the size of the resected specimen in this study. The result of our study revealed that the length of the greater curvature of the resected stomach was shorter in the EC-THS group compared to the IC-HHS group. In the EC-THS group, the length of the greater curvature tended to shorten as BMI increases. Resectable range of the proximal stomach might be limited in the EC-THS group as the degree of obesity increases. On the other hand, with regard to the transection at the lesser curvature side, it is possible to insert the tip of the linear stapler near the esophago-gastric junction. We supposed that this was the reason why there was no difference in the length of the resected lesser curvature. Despite of the higher proportion of middle third gastric cancer in the IC-HHS group, there was no difference in the proximal margin between the two groups. The intracorporeal procedure was superior not only in terms of intracorporeal reconstruction but also of intracorporeal transection, because it was possible to determine a more reliable transection level of the stomach to secure an optimal surgical margin. As described above, the extracorporeal anastomosis through a small incision sometimes requires excessive traction on the organs and increases intraoperative manipulation, especially in obese patients. The excessive traction of the organs in a narrow operative field sometimes causes unexpected vessel injuries in a blind spot. Additionally, although we carefully confirmed that there was no bleeding before making a small laparotomy, unexpected bleeding due to congestion of the stomach was often experienced at the time when the stomach was pulled out from the small laparotomy. This might cause the difference of intraoperative blood loss. In total laparoscopic gastrectomy, wide and anatomically undeviated surgical field minimizes unintended surgical trauma.

In conclusion, the IC-HHS technique for end-to-end Bi-I gastroduodenostomy revealed feasible with acceptable operation time and postoperative outcome. The DSLT and IC-HHS techniques allowed the operator to perform all procedures from initial skin incision to wound closure at the co-axial position without changing position. Although IC-HHS anastomosis requires an advanced suturing technique, we believe that it is necessary to keep basic training for intracorporeal suturing in addition to using automatic suturing equipment. Resectable range of the stomach was limited as the BMI increases in extracorporeal stomach transection. Intracorporeal stomach transection is desirable especially in obese patients. The advantage of TLDG has been reported providing a safe operative field during the reconstruction regardless of patient's figure. Additionally, the results of this study demonstrated another advantage of TLDG. It means that intracorporeal gastric resection makes it possible to perform safe gastric transection at a more proximal site in obese middle third gastric cancer patients without unexpected tissue damage.

\section{DECLARATIONS}

\section{Authors' contributions}

Conception and design of the study, data analysis, literature research, manuscript writing, manuscript editing, and manuscript revision: Ohmura Y, Suzuki H, Kotani K, Teramoto A 


\section{Availability of data and materials}

Not applicable.

\section{Financial support and sponsorship}

None.

\section{Conflicts of interest}

All authors declared that there are no conflicts of interest.

\section{Ethical approval and consent to participate}

All patients gave their written informed consent for laparoscopic operative procedures. This study protocol was approved by the institutional ethics committee.

\section{Consent for publication}

Not applicable.

\section{Copyright}

(c) The Author(s) 2019.

\section{REFERENCES}

1. Goh P, Tekant Y, Isaac J, Kum CK, Ngoi SS. The technique of laparoscopic Billroth II gastrectomy. Surg Laparosc Endosc 1992;2:25860 .

2. Kim W, Kim HH, Han SU, Kim MC, Hyung WJ, et al. Decreased morbidity of laparoscopic distal gastrectomy compared with open distal gastrectomy for stage I gastric cancer: short-term outcomes from a multicenter randomized controlled trial (KLASS-01). Ann Surg 2016;263:28-35.

3. Katia H, Mizusawa J, Katayama H, Takagi M, Yoshikawa T, et al. Short-term surgical outcomes from a phase III study of laparoscopyassisted versus open distal gastrectomy with nodal dissection for clinical stage IA/IB gastric cancer: Japan Clinical Oncology Group Study JCOG0912. Gastric Cancer 2017;20:699-708.

4. Bandoh T, Shiraishi N, Yamashita Y, Terachi T, Hashizume M, et al. Endoscopic surgery in Japan: the 12th national survey (2012-2013) by the Japan Society for Endoscopic Surgery. Asian J Endosc Surg 2017;10:345-53.

5. Deng Y, Zhang Y, Guo TK. Laparoscopy-assisted versus open distal gastrectomy for early gastric cancer: a meta-analysis based on seven randomized controlled trials. Surg Oncol 2015;24:71-7.

6. Fujisaki M, Shinohara T, Hanyu N, Kawano S, Tanaka Y, et al. Laparoscopic gastrectomy for gastric cancer in the elderly patients. Surg Endosc 2016;30:1380-7

7. Yoshida M, Koga S, Ishimaru K, Yamamoto Y, Matsuno Y, et al. Laparoscopy-assisted distal gastrectomy is feasible also for elderly patients aged 80 years and over: effectiveness and long-term prognosis. Surg Endosc 2017;31:4431-7.

8. Sugimoto M, Kinoshita T, Shibasaki H, Kato Y, Gotohda N, et al. Short-term outcome of total laparoscopic distal gastrectomy for overweight and obese patients with gastric cancer. Surg Endosc 2013;27:4291-6.

9. Son SY, Jung DH, Lee CM, Ahn SH, Ahn HS, et al. Laparoscopic gastrectomy versus open gastrectomy for gastric cancer in patients with body mass index of $30 \mathrm{~kg} / \mathrm{m} 2$ or more. Surg Endosc 2015;29:2126-32.

10. Kim CH, Song KY, Park CH, Seo YJ, Park SM, et al. A comparison of outcomes of three reconstruction methods after laparoscopic distal gastrectomy. J Gastric Cancer 2015;15:46-52.

11. Beyan C, Beyan E, Kaptan K, Ifran A, Uzar AI. Post-gastrectomy anemia: evaluation of 72 cases with post-gastrectomy anemia. Hematology 2007;12:81-4.

12. Kitano S, Iso Y, Moriyama M, Sugimachi K. Laparoscopy-assisted Billroth I gastrectomy. Surg Laparosc Endosc 1994;4:146-8.

13. Hiki N, Fukunaga T, Tokunaga M, Ohyama S, Yamada K, et al. An effective duodenum bulb mobilization for extracorporeal Billroth I anastomosis of laparoscopic gastrectomy. J Gastrointest Surg 2009;13:230-5.

14. Kim JJ, Song KY, Chin HM, Kim W, Jeon HM, et al. Totally laparoscopic gastrectomy with various types of intracorporeal anastomosis using laparoscopic linear staplers: preliminary experience. Surg Endosc 2008;22:436-42.

15. Ohmura Y, Nishi H, Fukuda K, Mano M. Technical modification of laparoscopy-assisted distal gastrectomy. J Jp Soc Endosc Surg 2004;9:191-5. (in Japanese)

16. Kanaya S, Gomi T, Momoi H, Tamaki N, Isobe H, et al. Delta-shaped anastomosis in totally laparoscopic Billroth I gastrectomy: new technique of intraabdominal gastroduodenostomy. J Am Coll Surg 2002;195:284-7.

17. Okabe H, Obama K, Tsunoda S, Tanaka E, Sakai Y. Advantage of completely laparoscopic gastrectomy with linear stapled reconstruction: a long-term follow-up study. Ann Surg 2014:259:109-16.

18. Kim JJ, Song KY, Chin HM, Kim W, Jeon HM, et al. Totally laparoscopic gastrectomy with various types of intracorporeal anastomosis using laparoscopic linear staplers: preliminary experience. Surg Endosc 2008;22:436-42.

19. Ohmura Y, Kumano K, Watanabe S, Mitsuoka N, Watanabe T. A new intracorporeal anastomosis method in laparoscopic distal 
gastrectomy. Billroth-I reconstruction by hemi-hand-sewn-technique. J Jp Soc Endosc Surg 2013;18:407. (in Japanese)

20. Dindo D, Demartines N, Clavien PA. Classification of surgical complications: a new proposal with evaluation in a cohort of 6336 patients and results of a survey. Ann Surg 2004;240:205-13.

21. Japanese Gastric Cancer Association. Japanese classification of gastric carcinoma: 3rd English edition. Gastric Cancer 2011;14:101-12.

22. Tanimura S, Higashino M, Fukunaga Y, Takemura M, Nishikawa T, et al. Intracorporeal Billroth 1 reconstruction by triangulating stapling technique after laparoscopic distal gastrectomy for gastric cancer. Surg Laparosc Endosc Percutan Tech 2008;18:54-8.

23. Ikeda T, Kawano H, Hisamatsu Y, Ando K, Saeki H, et al. Progression from laparoscopic-assisted to totally laparoscopic distal gastrectomy: comparison of circular stapler (i-DST) and linear stapler (BBT) for intracorporeal anastomosis. Surg Endosc 2013;27:325-32.

24. Nishimura S, Oki E, Tsutsumi S, Tsuda Y, Sugiyama M, et al. Clinical significance of totally laparoscopic distal gastrectomy: a comparison of short-term outcomes relative to open and laparoscopic-assisted distal gastrectomy. Surg Laparosc Endosc Percutan Tech 2016;26:372-6.

25. Byun C, Cui LH, Son SY, Hur H, Cho YK, et al. Linear-shaped gastroduodenostomy (LSGD): safe and feasible technique of intracorporeal Billroth I anastomosis. Surg Endosc 2016;30:4505-14.

26. Schneider C, Cobb W, Scott J, Carbonell A, Myers K, et al. Rapid excess weight loss following laparoscopic gastric bypass leads to increased risk of internal hernia. Surg Endosc 2011;25:1594-8.

27. Kim CH, Song KY, Park CH, Seo YJ, Park SM, et al. A comparison of outcomes of three reconstruction methods after laparoscopic distal gastrectomy. Gastric Cancer 2015;15:46-52.

28. Komatsu S, Ichikawa D, Kubota T, Okamoto K, Shiozaki A, et al. Clinical outcomes and quality of life according to types of reconstruction following laparoscopy-assisted distal gastrectomy for gastric cancer. Surg Laparosc Endosc Percutan Tech 2015;25:69-73.

29. Okabe H, Obama K, Tsunoda S, Tanaka E, Sakai Y. Advantage of completely laparoscopic gastrectomy with linear stapled reconstruction: a long-term follow-up study. Ann Surg 2014;259:109-16.

30. Takiguchi S, Sekimoto M, Miyake Y, Fujiwara Y, Yasuda T, et al. Totally laparoscopic distal gastrectomy using the hand-sewn Billroth-I anastomotic technique: report of a case. Surg Today 2003;33:371-4.

31. Chen K, Wu D, Pan Y, Cai JQ, Yan JF, et al. Totally laparoscopic gastrectomy using intracorporeally stapler or hand-sewn anastomosis for gastric cancer: a single-center experience of 478 consecutive cases and outcomes. World J Surg Oncol 2016;14:115.

32. Matsuo K, Shimura H, Tanaka S, Nakano M, Hashimoto T, et al. Laparoscopic distal gastrectomy with intracorporeal handsewn Billroth-I anastomosis (ICHSA). Surg Endosc 2012;26:2981-7.

33. Koeda K, Chiba T, Noda H, Nishinari Y, Segawa T, et al. Intracorporeal reconstruction after laparoscopic pylorus-preserving gastrectomy for middle-third early gastric cancer: a hybrid technique using linear stapler and manual suturing. Langenbecks Arch Surg 2016;401:397-402.

34. Sakata S, Kabir S, Petersen D, Doudle M, Stevenson AR. Are we burying our heads in the sand? Preventing small bowel obstruction

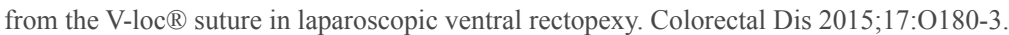

35. Filser J, Reibetanz J, Krajinovic K, Germer CT, Dietz UA, et al. Small bowel volvulus after transabdominal preperitoneal hernia repair due to improper use of V-Loc barbed absorbable wire - do we always "read the instructions first"? Int J Surg Case Rep 2015;8:193-5.

36. Donnellan NM, Mansuria SM. Small bowel obstruction resulting from laparoscopic vaginal cuff closure with a barbed suture. J Minim Invasive Gynecol 2011;18:528-30.

37. Ser KH, Lee WJ, Lee YC, Chen JC, Su YH, et al. Experience in laparoscopic sleeve gastrectomy for morbidly obese Taiwanese: stapleline reinforcement is important for preventing leakage. Surg Endosc 2010;24:2253-9.

38. Inoue K, Michiura T, Fukui J, Mukaide H, Ozaki T, et al. Staple-line reinforcement of the duodenal stump with intracorporeal Lembert's sutures in laparoscopic distal gastrectomy with Roux-en-Y reconstruction for gastric cancer. Surg Laparosc Endosc Percutan Tech 2016;26:338-42.

39. Kim MC, Kim SY, Kim KW. Laparoscopic reinforcement suture (LARS) on staple line of duodenal stump using barbed suture in laparoscopic gastrectomy for gastric cancer: a prospective single arm phase II study. J Gastric Cancer 2017;17:354-62.

40. Kim MG, Kawada H, Kim BS, Kim TH, Kim KC, et al. A totally laparoscopic distal gastrectomy with gastroduodenostomy (TLDG) for improvement of the early surgical outcomes in high BMI patients. Surg Endosc 2011;25:1076-82.

41. Lee HH, Song KY, Lee JS, Park SM, Kim JJ. Delta-shaped anastomosis, a good substitute for conventional Billroth I technique with comparable long-term functional outcome in totally laparoscopic distal gastrectomy. Surg Endosc 2015;29:2545-52.

42. Jeong O, Jung MR, Park YK, Ryu SY. Safety and feasibility during the initial learning process of intracorporeal Billroth I (delta-shaped) anastomosis for laparoscopic distal gastrectomy. Surg Endosc 2015;29:1522-9.

43. Park KB, Kwon OK, Yu W, Jang BC. Body composition changes after totally laparoscopic distal gastrectomy with delta-shaped anastomosis: a comparison with conventional Billroth I anastomosis. Surg Endosc 2016;30:4286-93. 